\title{
Adaptive output feedback neural network control of uncertain non-affine systems with unknown control direction
}

\author{
Mohammad M. Arefi ${ }^{\mathrm{a}}$, Jafar Zarei ${ }^{\mathrm{b}}$, Hamid R. Karimi ${ }^{\mathrm{c}, *}$ \\ ${ }^{a}$ Department of Power and Control Engineering, School of Electrical and Computer Engineering, Shiraz University, \\ ${ }^{\mathrm{b}}$ Department Control Engineering, School of Electrical and Electronics Engineering, Shiraz University of Technology, \\ 71557-13876 Shiraz, Iran \\ ${ }^{\mathrm{c}}$ Department of Engineering, Faculty of Engineering and Science, University of Agder, N-4898 Grimstad, Norway
}

Received 22 February 2014; received in revised form 24 April 2014; accepted 19 May 2014

Available online 28 May 2014

\begin{abstract}
This paper deals with the problem of adaptive output feedback neural network controller design for a SISO non-affine nonlinear system. Since in practice all system states are not available in output measurement, an observer is designed to estimate these states. In comparison with the existing approaches, the current method does not require any information about the sign of control gain. In order to handle the unknown sign of the control direction, the Nussbaum-type function is utilized. In order to approximate the unknown nonlinear function, neural network is firstly exploited, and then to compensate the approximation error and external disturbance a robustifying term is employed. The proposed controller is designed based on strict-positive-real (SPR) Lyapunov stability theory to ensure the asymptotic stability of the closed-loop system. Finally, two simulation studies are presented to demonstrate the effectiveness of the developed scheme.
\end{abstract}

Keywords: Observer-based control; Uncertain non-affine systems; Unknown gain direction; Neural network control

*Corresponding author. Tel.: +47 37233000.

E-mail addresses:

arefi@shirazu.ac.ir (M.M. Arefi), zarei@sutech.ac.ir (J. Zarei), hamid.r.karimi@uia.no (H.R. Karimi). 


\section{Introduction}

Robust control of nonlinear systems is considered as a challenging problem in control theory and has attracted a lot of devotion in the past decades [1]. However, most of the previous works are based on some idealized assumptions, one of which is that the dynamics of the system is completely matched with its mathematical model [2]. There are, of course, unavoidable model uncertainties for any practical system, which lead the degradation of controller performance [3]. In these cases, the conventional control approaches are not applicable, and usually the universal functions approximators (UFAs)-based adaptive approaches are suggested to address this issue [4-8]. Therefore, the analytical study of adaptive control of uncertain nonlinear systems using UFA has received much attention during last decade.

In this regards, neural networks (NNs) and fuzzy logic (FL) are usually exploited to approximate the unknown uncertainties [9-13]. Then, adaptive laws are designed to adjust the parameters of the NNs and FL. To this end, two different approaches are used to design the controller: direct and indirect approaches [14-18]. The main drawback of indirect approaches is singularity problem in calculating the estimation of the control gain direction [19]. The projection algorithm, and using regularized inverse of control gain direction are two main strategies to deal with the singularity problem [20,21]. In current study, an indirect adaptive controller approach using $\mathrm{NN}$ is proposed to overcome aforementioned problem.

The main drawback of the UFAs-based controller design is that it requires to know the sign of the control gain matrix in advance; however, this is not generally a realistic assumption [19]. The problem is much more challenging in cases that the sign of control gain is not known a priori. To address this issue, Nussbaum-gain technique can be used [22-24]. For instance, adaptive fuzzy controller is developed for uncertain MIMO, and SISO nonlinear systems with unknown control gain directions in [7], and [8], respectively. However, the states of the system are assumed to be available in most of the previous works [9], while this assumption may not be satisfied in practical applications. In these cases, observer-based or output feedback controllers might be used [14,2530]. For instance, a Linear Matrix Inequality (LMI) strategy to design

static output-feedback controllers has been presented in [25]. In [26], an observer-based $\mathrm{H} \infty$ controller has been developed for Markovian jump systems considering actuator saturation

nonlinearity and time-delay. Besides, output feedback controller has been proposed for Markov jump systems with passivity criteria subjected to time delays [27]. In addition, several approaches have been recently developed for nonlinear non-affine systems $[4,31,32]$. In these methods, the unknown non-affine function is firstly converted into affine-like form using the Mean Value Theorem. In this regards, an adaptive fuzzy controller using state-feedback for a SISO non-affine system with unknown gain sign is presented [24].

In current work, an observer-based adaptive NN controller is developed for an uncertain nonlinear

non-affine system with unknown gain sign. The main innovations of the paper are three folds:

(i) The Nussbaum-gain technique is effectively employed to design an adaptive controller for a SISO non-affine system with unmeasurable states. The proposed method can also be applied to affine systems.

(ii) Using indirect adaptive control and Mean Value Theorem enable us to avoid the controller singularity issue.

(iii) The effects of approximation error in NN and external disturbance are compensated using a robustifying term in the control signal, so that the proposed approach is able to handle a certain degree of uncertainties. 
The remained part of this paper is structured as follows: The problem formulation is presented in Section 2. A design procedure for observer-based adaptive NN control is established in Section 3. The promising results of the presented method are demonstrated through simulation results in Section 4. Finally, the paper is concluded in Section 5.

\section{Problem statement}

Consider a SISO uncertain nonlinear system represented with the following state-space equations:

$$
\begin{aligned}
& \dot{x}_{i}=x_{i+1}, \quad i=1,2, \ldots, n-1 \\
& \dot{x}_{n}=f(\mathbf{x}, u)+d(t) \\
= & { }_{1} y \quad x
\end{aligned}
$$

(1) where

$\mathbf{x} \in R^{n}$ is the plant state vector, $u \in R$ is the control input of the system, $y \in R$ is the output,

$f(\mathbf{x}, u)$ is an unknown smooth non-affine function in control input and the state vector, and ( $d t \quad R$ is the externaldisturbance. The goal is to design a controller that the system output track ) $\in$ the reference signal $y_{m}$.

Assumption 1. For all $(\mathbf{x}, u) \in \Omega_{\mathbf{x}} \times \mathfrak{R}$ with a controllability region $\Omega_{\mathbf{x}}$, the function $g(\mathbf{x}, u)=(\partial f$ $(\mathbf{x}, u) / \partial u) \neq 0$ is nonzero.

Assumption 2. The reference signal $y_{m}$ and its $n$ derivatives, i.e. $y_{m}^{i}, \quad i=1, \ldots, n$, are bounded, smooth, and accessible for design purpose.

Remark 1. Some physical plants could be represented with model (1). For example, second order mechanical systems like magnetic suspension system or some chaotic systems like DuffingHolmes system may be described by Eq. (1).

Remark 2. It is usually assumed that the sign of function $g(\mathbf{x}, u)$ is known in advance $[31,32]$. Nevertheless, Assumption 1 indicates that it is not required to know the gain sign of function $g(\mathbf{x}, u)$. In this paper, to deal with this problem the Nussbaum type function is utilized. Definition 1. [22]. A function $N(\varsigma)$ is called Nussbaum type function if it meets the following
properties:

$$
\begin{aligned}
& \lim _{z \rightarrow \infty} \sup \frac{1}{z} \int_{0}^{z} N(\varsigma) d \varsigma=+\infty \\
& \lim _{z \rightarrow \infty} \inf \frac{1}{z} \int_{0}^{z} N(\varsigma) d \varsigma=-\infty
\end{aligned}
$$

Frequently used Nussbaum type functions are $\varsigma^{2} \cos (\varsigma), \varsigma^{2} \sin (\varsigma)$, and $\exp \left(\varsigma^{2}\right) \cos (\pi \varsigma / 2)$. The even Nussbaum function $N(\varsigma)=\exp \left(\varsigma^{2}\right) \cos (\pi \varsigma / 2)$ is exploited in this paper.

Lemma 1. [33]. Suppose $V(t), \varsigma(t)$ are functions defined on $\left[0, t_{f}\right)$ with $V(t)>0, \quad \forall t \in\left[0, t_{f}\right)$, and $N(\varsigma)$ is an even smooth Nussbaum-type function. If the following inequality holds:

$$
V(t) \leq c_{0} \pm \int_{0}^{t}(g N(\varsigma) \pm 1) \dot{\varsigma} d \tau
$$

where $c_{0}$ is a proper constant, and $g$ represents a non-zero constant, then $V(t), \varsigma(t)$, and $\int_{0}^{t}(g N(\varsigma) \pm 1) \dot{\zeta} d \tau$ have to be bounded on $\left[0, t_{f}\right)$. 
Lemma 2. (Mean Value Theorem): Suppose $f(x, y): \mathfrak{R}^{n} \times \mathfrak{R} \rightarrow \mathfrak{R}$ is continuous at both endpoints $y=a$ and $y=b$, and has a derivative at each point of an open set $\Re^{n} \times(a, b)$. Therefore, there is a point $\pi \in(a, b)$ in a way that [4]

$$
f(x, b)-f(x, a)=f^{\prime}(x, \pi)(b-a)
$$

The tracking error is defined as

$$
e_{1}=y-y_{m} .
$$

Indicating

$$
\begin{aligned}
& \mathbf{x}=\left[x_{1}, \ldots, x_{1}^{(n-1)}\right]^{T} \\
& \mathbf{y}_{m}=\left[y_{m}, \ldots, y_{m}^{(n-1)}\right]^{T}
\end{aligned}
$$

then

$$
\mathbf{e}=\mathbf{x}-\mathbf{y}_{m}=\left[e_{1}, \ldots, e_{1}^{n-1}\right]^{T} .
$$

Considering the reference model of the form $\dot{\mathbf{y}}_{m}=\mathbf{A} \mathbf{y}_{m}+\mathbf{B} y_{m}^{n}$, the tracking error system can be written as

$$
\begin{aligned}
& \dot{\mathbf{e}}=\mathbf{A e}+\mathbf{B}\left[f(\mathbf{x}, u)-y_{m}^{n}+d(t)\right] \\
& e_{1}=\mathbf{C}^{T} \mathbf{e}
\end{aligned}
$$

where

$$
\begin{aligned}
\mathbf{A} & =\left[\begin{array}{cccc}
0 & 1 & \ldots & 0 \\
0 & 0 & \ldots & 0 \\
\vdots & \vdots & \ldots & \vdots \\
0 & 0 & \ldots & 1 \\
0 & 0 & \ldots & 0
\end{array}\right]_{n \times n} \\
\mathbf{B} & =\left[\begin{array}{c}
0 \\
0 \\
\vdots \\
0 \\
1
\end{array}\right]_{n \times 1}, \quad \mathbf{C}^{\mathrm{T}}=[1,0, \ldots, 0]_{1 \times n} .
\end{aligned}
$$

Considering the Mean Value Theorem, one can write

$$
f(\mathbf{x}, u)=f(\mathbf{x})+g\left(\mathbf{x}, u^{*}\right) u
$$

where $u^{*}$ is a point between zero and $u$. Hence, Eq. (9) can be rewritten as

$$
\begin{aligned}
& \dot{\mathbf{e}}=\mathbf{A e}+\mathbf{B}\left[f(\mathbf{x})+g\left(\mathbf{x}, u^{*}\right) u-y_{m}^{n}+d(t)\right] \\
& e_{1}=\mathbf{C}^{T} \mathbf{e}
\end{aligned}
$$

Suppose $f(\mathbf{x}), g\left(\mathbf{x}, u^{*}\right)$ are known, $d(t)=\mathbf{0}$, and the state $\mathbf{x}$ are available, then, from Eq. (11) the ideal controller can be chosen as

$$
u_{\text {ideal }}=g^{-1}\left(\mathbf{x}, u^{*}\right)\left[-f(\mathbf{x})+y_{m}^{n}-\mathbf{K}_{c}^{T} \mathbf{e}\right]
$$

where $\mathbf{K}_{c}^{T}$ is the feedback gain vector to ensure the characteristic polynomial of $\mathbf{A}-\mathbf{B K}_{c}^{T}$ Hurwitz. Consequently, it can be shown that $\lim _{t \rightarrow \infty} \mathbf{e}(t)=\mathbf{0}$. Nevertheless, $f(\mathbf{x})$ and $g\left(\mathbf{x}, u^{*}\right)$ are unknown and the systems states $\mathbf{x}$ are not measurable, so the controller $u_{\text {ideal }}$ cannot be realized. 
A comprehensive solution is to employ NNs to approximate unknown functions and design an observer to estimate the system states $\mathbf{x}$. It is shown that the nonlinear continuous functions can be approximated by NNs with an arbitrary accuracy.

\section{Adaptive neural control design}

Since the state vector is unmeasurable in practice, it cannot be used in the controller design. Therefore, the unmeasurable states are firstly estimated. The following structure is considered for the observer:

$$
\begin{aligned}
& \dot{\hat{\mathbf{e}}}=\mathbf{A} \hat{\mathbf{e}}-\mathbf{B} \mathbf{K}_{c}^{T} \hat{\mathbf{e}}+\mathbf{K}_{o}\left(e_{1}-\hat{e}_{1}\right) \\
& \hat{e}_{1}=\mathbf{C}^{T} \hat{e}
\end{aligned}
$$

where $\mathbf{K}_{o}$ is the observer gain vector to be designed so that the matrix $\mathbf{A}-\mathbf{K}_{o} \mathbf{C}^{T}$ is Hurwitz, and $\hat{\mathbf{e}}$ is the estimation of $\mathbf{e}$. Define the observation error as $\tilde{\mathbf{e}}=\mathbf{e}-\hat{\mathbf{e}}$. Then using Eqs. (11) and (12) one can write

$$
\begin{aligned}
& \dot{\tilde{\mathbf{e}}}=\left(\mathbf{A}-\mathbf{K}_{o} \mathbf{C}^{T}\right) \tilde{\mathbf{e}}+\mathbf{B}\left[f(\mathbf{x})-y_{m}^{n}+\mathbf{K}_{c}^{T} \hat{\mathbf{e}}+d(t)+g\left(\mathbf{x}, u^{*}\right) u\right] \\
& \tilde{e}_{1}=\mathbf{C}^{T} \tilde{\mathbf{e}}
\end{aligned}
$$

or, equivalently,

$$
\begin{aligned}
& \dot{\tilde{\mathbf{e}}}=\left(\mathbf{A}-\mathbf{K}_{o} \mathbf{C}^{T}\right) \tilde{\mathbf{e}}+\mathbf{B}\left[f(\mathbf{x})-y_{m}^{n}+\mathbf{K}_{c}^{T} \hat{\mathbf{e}}+d(t)+\left(g\left(\mathbf{x}, u^{*}\right)+g_{0}\right) u-g_{0} u\right] \\
& \tilde{e}_{1}=\mathbf{C}^{T} \tilde{\mathbf{e}}
\end{aligned}
$$

where $g_{0}$ is an arbitrary nonzero constant. Defining $f_{1}(\overline{\mathbf{x}})=f(\mathbf{x})+\left(g\left(\mathbf{x}, u^{*}\right)+g_{0}\right) u$, Eq. (14) can be rewritten as

$$
\begin{aligned}
& \dot{\tilde{\mathbf{e}}}=\left(\mathbf{A}-\mathbf{K}_{o} \mathbf{C}^{T}\right) \tilde{\mathbf{e}}+\mathbf{B}\left[f_{1}(\overline{\mathbf{x}})-y_{m}^{n}+\mathbf{K}_{c}^{T} \hat{\mathbf{e}}+d(t)-g_{0} u\right] \\
& \tilde{e}_{1}=\mathbf{C}^{T} \tilde{\mathbf{e}}
\end{aligned}
$$

where $\overline{\mathbf{x}}=\left[\mathbf{x}^{T}, u\right]^{T}$. Thereafter, estimation of $f_{1}(\overline{\mathbf{x}})$ obtained from a NN is utilized to design an adaptive controller. The adaptation law is designed so that the controller meets the predefined objectives and guarantees boundedness of all signals in the closed-loop system. The strictpositive-real (SPR) Lyapunov design approach is used to guarantee the stability of the closedloop system as $\tilde{e}_{1}$ in Eq. (15) is accessible. The dynamics of output error in Eq. (15) can be obtained as

$$
\tilde{e}_{1}=H(s)\left[f_{1}(\overline{\mathbf{x}})-y_{m}^{n}+\mathbf{K}_{c}^{T} \hat{\mathbf{e}}+d(t)-g_{0} u\right]
$$

where

$$
H(s)=\mathbf{C}^{T}\left(s \mathbf{I}-\left(\mathbf{A}-\mathbf{K}_{o} \mathbf{C}^{T}\right)\right)^{-1} \mathbf{B}
$$

is a known stable transfer function. Eq. (16) is written as

$$
\tilde{e}_{1}=H(s) L(s)\left[L^{-1}(s) f_{1}(\overline{\mathbf{x}})+L^{-1}(s)\left(\mathbf{K}_{c}^{T} \hat{\mathbf{e}}-y_{m}^{n}\right)+L^{-1}(s) d(t)-L^{-1}(s) g_{0} u\right]
$$

with

$$
L(s)=s^{m}+b_{1} s^{m-1}+\cdots+b_{m}(m<n)
$$

to be used in the SPR-Lyapunov design procedure.

Note that $L(s)$ should be chosen such that $L^{-1}(s)$ be a suitable stable transfer function and $H(s) L(s)$ be a proper SPR transfer function. Therefore, the state-space realization of Eq. (18) can 
be stated as

$$
\begin{aligned}
& \dot{\tilde{\mathbf{e}}}=\left(\mathbf{A}-\mathbf{K}_{o} \mathbf{C}^{T}\right) \tilde{\mathbf{e}}+\mathbf{B}_{c}\left[L^{-1}(s) f_{1}(\overline{\mathbf{x}})+L^{-1}(s)\left(\mathbf{K}_{c}^{T} \hat{\mathbf{e}}-y_{m}^{n}\right)+L^{-1}(s) d(t)-L^{-1}(s) g_{0} u\right] \\
& \tilde{e}_{1}=\mathbf{C}^{T} \tilde{\mathbf{e}}
\end{aligned}
$$

with SPR transfer function as

$$
H_{c}(s)=\mathbf{C}^{T}\left(s \mathbf{I}-\left(\mathbf{A}-\mathbf{K}_{o} \mathbf{C}^{T}\right)\right)^{-1} \mathbf{B}_{c}
$$

Remark 3. There is no unique choice for the input arguments of unknown function $f_{1}(\overline{\mathbf{x}})$, i.e. $\overline{\mathbf{x}}$. Actually, since $u$ is a function of states $\mathbf{x}$ and $\mathbf{y}_{m}^{n}$, and $\mathbf{y}_{m}^{n}$ is also bounded, then it can quite simply be shown that $\overline{\mathbf{x}}$ is a function of $\mathbf{x}$.

In order to approximate unknown function $f_{1}(\overline{\mathbf{x}})$, an RBF neural network with two layers, and $l$ nodes are used as

$$
\hat{f}_{1}(\hat{\mathbf{x}} \mid \boldsymbol{\Theta})=\boldsymbol{\Theta} \xi(\hat{\mathbf{x}})
$$

where

$\boldsymbol{\Theta}=\left[\begin{array}{llll}\theta_{11} & \theta_{12} & \cdots & \theta_{1 l}\end{array}\right]$ is the adjustable parameter vector of the $\mathrm{NN}$, and $\xi(\hat{\mathbf{x}})=\left[\xi_{1}(\hat{\mathbf{x}}), \ldots, \xi_{l}(\hat{\mathbf{x}})\right]^{\mathbf{T}}$ is a radial basis function vector that is fixed a priori. The commonly used Gaussian functions are used as basis function

$$
\xi_{i}(\mathbf{x})=\exp \left(-\frac{\left\|\mathbf{x}-\boldsymbol{\mu}_{i}\right\|^{2}}{\eta_{i}^{2}}\right), \quad i=1,2, \ldots, l
$$

where $\eta_{i}$ is the width, and $\boldsymbol{\mu}_{i}=\left[\mu_{i 1}, \mu_{i 1}, \ldots, \mu_{i n}\right]^{T}$ is the center vector of the Gaussian function.

It is supposed that $\mathbf{x}, \hat{\mathbf{x}}$, and $\boldsymbol{\Theta}$ belong to compact sets $U_{1}, U_{2}$, and $\Omega$, respectively, defined as

$$
\begin{aligned}
& U_{1}=\left\{\mathbf{x} \in \mathfrak{R}^{n}:\|\mathbf{x}\| \leq M_{1}\right\} \\
& U_{2}=\left\{\hat{\mathrm{x}} \in \mathfrak{R}^{n}:\|\hat{\mathbf{x}}\| \leq M_{2}\right\} \\
& \Omega=\left\{\boldsymbol{\Theta} \in \mathfrak{R}^{l}:\|\boldsymbol{\Theta}\| \leq M_{3}\right\}
\end{aligned}
$$

where $M_{1}, M_{2}$, and $M_{3}$ are the designed parameters.

Define the optimal parameter vector $\boldsymbol{\Theta}^{*}$

$$
\boldsymbol{\Theta}^{*}=\arg \min _{\hat{\boldsymbol{\Theta}} \in \Omega}\left\{\sup _{\mathbf{x} \in U_{1}, \hat{\mathbf{x}} \in U_{2}}\left|f_{1}(\overline{\mathbf{x}})-\hat{f}_{1}(\hat{\mathbf{x}} \mid \boldsymbol{\Theta})\right|\right\}
$$

and $\mathrm{NN}$ approximation error $\delta_{n}$

$$
\delta_{n}(\mathbf{x}, \hat{\mathbf{x}})=f_{1}(\overline{\mathbf{x}})-\hat{f}_{1}\left(\hat{\mathbf{x}} \mid \Theta^{*}\right)
$$

Here, $\boldsymbol{\Theta}$ denotes the estimation of $\boldsymbol{\Theta}^{*}$ and let $\tilde{\boldsymbol{\Theta}}=\boldsymbol{\Theta}-\boldsymbol{\Theta}^{*}$.

Remark 4. Since $\left(\mathbf{A}-\mathbf{B} \mathbf{K}_{c}^{T}\right)$ is Hurwitz, the following Lyapunov equation has a unique positive-definite solution $\mathbf{P}_{1}$ for any given symmetric positive-definite matrix $\mathbf{Q}_{1}$

$$
\left(\mathbf{A}-\mathbf{B} \mathbf{K}_{c}^{T}\right)^{T} \mathbf{P}_{1}+\mathbf{P}_{1}\left(\mathbf{A}-\mathbf{B} \mathbf{K}_{c}^{T}\right)+\mathbf{Q}_{1}=\mathbf{0}
$$


Assumption 3. For the given positive-definite matrix $\mathbf{Q}_{2}$, there exists a positive-definite solution $\mathbf{P}_{2}$ for the matrix equations

$$
\begin{gathered}
\left(\mathbf{A}-\mathbf{K}_{o} \mathbf{C}^{T}\right)^{T} \mathbf{P}_{2}+\mathbf{P}_{2}\left(\mathbf{A}-\mathbf{K}_{o} \mathbf{C}^{T}\right)+\mathbf{Q}_{2}=\mathbf{0} \\
\mathbf{P}_{2} \mathbf{B}_{c}=\mathbf{C}
\end{gathered}
$$

The adaptive NN-based controller is designed as

$$
u=N(\varsigma)\left[-\boldsymbol{\Theta} \xi(\hat{\mathbf{x}})+y_{m}^{n}-\mathbf{K}_{\mathrm{c}}^{\mathrm{T}} \hat{\mathbf{e}}+u_{a}+u_{s}\right]
$$

with

$$
\begin{aligned}
& u_{a}=-\mathbf{K}_{o}^{T} \mathbf{P}_{1} \hat{\mathbf{e}} \\
& u_{s}=-k \operatorname{sgn}\left(\mathbf{B}_{c}^{T} \mathbf{P}_{2} \tilde{\mathbf{e}}\right) \\
& \dot{\boldsymbol{\zeta}}=\tilde{e}_{1}^{T} L^{-1}(s)\left[\boldsymbol{\Theta} \xi(\hat{\mathbf{x}})-y_{m}^{n}+\mathbf{K}_{c}^{T} \hat{\mathbf{e}}-u_{a}-u_{s}\right]
\end{aligned}
$$

and the following adaptation law

$$
\dot{\boldsymbol{\Theta}}=\gamma \mathbf{B}_{c}^{T} \mathbf{P}_{2} \tilde{\mathbf{e}} L^{-1}(s) \xi^{T}(\hat{\mathbf{x}})
$$

where $u_{a}$ is the feedback controller for $\hat{\mathbf{e}}, u_{s}$ should be designed such that both NN approximation error and external disturbances are compensated, and $\gamma>0$ is the adaptation gain that should be designed.

Assumption 4. $\delta_{m}=L^{-1}(s)\left(\delta_{n}(\mathbf{x}, \hat{\mathbf{x}})+d(t)+u_{a}+u_{s}\right)-u_{a}-u_{s}$ is bounded. In other words, $\delta_{m}$ satisfies $\left\|\delta_{m}\right\|<\delta$.

Concerning to the above discussions, the following theorem summarizes the main property of observer-based adaptive neural network controller.

Theorem 1. For the system defined by Eq. (1), the adaptive nonlinear controller is considered as Eqs. (29)-(30) with the adaptation law (31). If Assumptions 1-4 are held, the following properties are guaranteed using the proposed scheme:

(1) The whole closed-loop system is stable, i.e., $\mathbf{x}, \hat{\mathbf{x}}, \mathbf{e}, \hat{\mathbf{e}}, u \in L_{\infty}$

(2) $\lim _{t \rightarrow \infty} e_{1}=0$

Proof. Define the following Lyapunov function candidate

$$
V=\frac{1}{2} \hat{\mathbf{e}}^{\mathbf{T}} \mathbf{P}_{1} \hat{\mathbf{e}}+\frac{1}{2} \tilde{\mathbf{e}}^{\mathbf{T}} \mathbf{P}_{2} \tilde{\mathbf{e}}+\frac{1}{2 \gamma} \tilde{\boldsymbol{\Theta}} \tilde{\boldsymbol{\Theta}}^{T}
$$

The derivative of $V$ is

$$
\dot{V}=\frac{1}{2} \dot{\hat{\mathbf{e}}}^{T} \mathbf{P}_{1} \hat{\mathbf{e}}+\frac{1}{2} \hat{\mathbf{e}}^{T} \mathbf{P}_{1} \dot{\hat{\mathbf{e}}}+\frac{1}{2} \dot{\tilde{\mathbf{e}}}^{T} \mathbf{P}_{2} \tilde{\mathbf{e}}+\frac{1}{2} \tilde{\mathbf{e}}^{T} \mathbf{P}_{2} \dot{\tilde{\mathbf{e}}}+\frac{1}{\gamma} \tilde{\mathbf{\Theta}} \dot{\tilde{\mathbf{\Theta}}}^{T}
$$

Substituting Eqs. (12) and (20) into Eq. (33), one can write

$$
\begin{aligned}
\dot{V}= & \frac{1}{2} \hat{\mathbf{e}}^{\mathbf{T}}\left[\left(\mathbf{A}-\mathbf{B} \mathbf{K}_{c}^{T}\right)^{T} \mathbf{P}_{1}+\mathbf{P}_{1}\left(\mathbf{A}-\mathbf{B} \mathbf{K}_{c}^{T}\right)\right] \hat{\mathbf{e}}+\hat{\mathbf{e}}^{T} \mathbf{P}_{1} \mathbf{K}_{o} \mathbf{C}^{T} \tilde{\mathbf{e}} \\
& +\frac{1}{2} \tilde{\mathbf{e}}^{T}\left[\left(\mathbf{A}-\mathbf{K}_{o} \mathbf{C}^{T}\right)^{T} \mathbf{P}_{2}+\mathbf{P}_{2}\left(\mathbf{A}-\mathbf{K}_{o} \mathbf{C}^{T}\right)\right] \tilde{\mathbf{e}}
\end{aligned}
$$




$$
\begin{aligned}
& +\tilde{\mathbf{e}}^{T} \mathbf{P}_{2} \mathbf{B}_{c} L^{-1}(s) f_{1}(\overline{\mathbf{x}})+L^{-1}(s)\left(\mathbf{K}_{c}^{T} \hat{\mathbf{e}}-y_{m}^{n}\right) \\
& \left.+L^{-1}(s) d(t)-L^{-1}(s) g_{0} u\right]+\frac{1}{\gamma} \tilde{\mathbf{\Theta}} \dot{\tilde{\Theta}}^{T}
\end{aligned}
$$

Then using Eqs. (27) and (28), Eq. (34) can be rewritten as

$$
\begin{aligned}
\dot{V}= & -\frac{1}{2} \hat{\mathbf{e}}^{T} \mathbf{Q}_{1} \hat{\mathbf{e}}+\hat{\mathbf{e}}^{T} \mathbf{P}_{1} \mathbf{K}_{o} \mathbf{C}^{T} \tilde{\mathbf{e}}-\frac{1}{2} \tilde{\mathbf{e}}^{T} \mathbf{Q}_{2} \tilde{\mathbf{e}} \\
& +\tilde{\mathbf{e}}^{T} \mathbf{P}_{2} \mathbf{B}_{c}\left[L^{-1}(s) f_{1}(\overline{\mathbf{x}})+L^{-1}(s)\left(\mathbf{K}_{c}^{T} \hat{\mathbf{e}}-y_{m}^{n}\right)\right. \\
& \left.+L^{-1}(s) d(t)-L^{-1}(s) g_{0} u\right]+\frac{1}{\gamma} \tilde{\boldsymbol{\Theta}} \dot{\tilde{\boldsymbol{\Theta}}}^{T}
\end{aligned}
$$

By using Eqs. (22) and (26), Eq. (35) can be rewritten as

$$
\begin{aligned}
\dot{V}= & -\frac{1}{2} \hat{\mathbf{e}}^{T} \mathbf{Q}_{1} \hat{\mathbf{e}}+\hat{\mathbf{e}}^{T} \mathbf{P}_{1} \mathbf{K}_{o} \mathbf{C}^{T} \tilde{\mathbf{e}}-\frac{1}{2} \tilde{\mathbf{e}}^{T} \mathbf{Q}_{2} \tilde{\mathbf{e}} \\
& +\tilde{\mathbf{e}}^{T} \mathbf{P}_{2} \mathbf{B}_{c}\left[-L^{-1}(s) \tilde{\mathbf{\Theta}} \xi(\hat{\mathbf{x}})+L^{-1}(s) \boldsymbol{\Theta} \xi(\hat{\mathbf{x}})\right. \\
& +L^{-1}(s) \delta_{f}(\mathbf{x}, \hat{\mathbf{x}})+\left(u_{a}+u_{s}\right)-\left(u_{a}+u_{s}\right) \\
& \left.+L^{-1}(s)\left(\mathbf{K}_{c}^{T} \hat{e}-y_{m}^{n}\right)+L^{-1}(s) d(t)-L^{-1}(s) g_{0} u\right]+\frac{1}{\gamma} \tilde{\mathbf{\Theta}} \dot{\tilde{\mathbf{\Theta}}}^{T}
\end{aligned}
$$

Substituting Eqs. (29) and (30) and adaptation law (31) in Eq. (36) and then using Assumption 4 one can write

$$
\begin{aligned}
\dot{V}= & -\frac{1}{2} \hat{\mathbf{e}}^{T} \mathbf{Q}_{1} \hat{\mathbf{e}}-\frac{1}{2} \tilde{\mathbf{e}}^{T} \mathbf{Q}_{2} \tilde{\mathbf{e}}+\tilde{\mathbf{e}}^{\mathbf{T}} \mathbf{P}_{2} \mathbf{B}_{c} \delta_{m}+\tilde{\mathbf{e}}^{\mathbf{T}} \mathbf{P}_{2} \mathbf{B}_{c} u_{s} \\
& +\tilde{\mathbf{e}}^{\mathbf{T}} \mathbf{P}_{2} \mathbf{B}_{c} L^{-1}(s)\left(\boldsymbol{\Theta} \xi(\hat{\mathbf{x}})-y_{m}^{n}+\mathbf{K}_{c}^{T} \hat{\boldsymbol{e}}-u_{a}-u_{s}\right)\left(1+g_{0} N(\varsigma)\right) \\
= & -\frac{1}{2} \hat{\mathbf{e}}^{T} \mathbf{Q}_{1} \hat{\mathbf{e}}-\frac{1}{2} \tilde{\mathbf{e}}^{T} \mathbf{Q}_{2} \tilde{\mathbf{e}}+\tilde{\mathbf{e}}^{\mathbf{T}} \mathbf{P}_{2} \mathbf{B}_{c}\left(\delta_{m}+u_{s}\right)+\left(1+g_{0} N(\varsigma)\right) \dot{\boldsymbol{\zeta}}
\end{aligned}
$$

Substituting $u_{s}$ into Eq. (37) yields:

$$
\dot{V}=-\frac{1}{2} \hat{\mathbf{e}}^{T} \mathbf{Q}_{1} \hat{\mathbf{e}}-\frac{1}{2} \tilde{\mathbf{e}}^{T} \mathbf{Q}_{2} \tilde{\mathbf{e}}+(\delta-k)\left|\left(\tilde{\mathbf{e}}^{\mathrm{T}} \mathbf{P}_{2} \mathbf{B}_{c}\right)\right|+\left(1+g_{0} N(\varsigma)\right) \dot{\boldsymbol{\zeta}}
$$

Selecting $k \geq \delta$, we have

$$
\dot{V} \leq-\frac{1}{2} \hat{\mathbf{e}}^{T} \mathbf{Q}_{1} \hat{\mathbf{e}}-\frac{1}{2} \tilde{\mathbf{e}}^{T} \mathbf{Q}_{2} \tilde{\mathbf{e}}+\left(1+g_{0} N(\varsigma)\right) \dot{\zeta}
$$

Considering $\mathbf{Q}=\operatorname{diag}\left[\mathbf{Q}_{1}, \mathbf{Q}_{2}\right]$ and $\mathbf{E}^{T}=\left[\hat{\mathbf{e}}^{T}, \tilde{\mathbf{e}}^{T}\right]$, Eq. (39) becomes

$$
\dot{V} \leq-\frac{1}{2} \mathbf{E}^{T} \mathbf{Q E}+\left(1+g_{0} N(\varsigma)\right) \dot{\zeta}
$$

Keeping in mind that $-\mathbf{E}^{\mathbf{T}} \mathbf{Q E} \leq-\lambda_{\min }(\mathbf{Q})\|\mathbf{E}\|^{2}$, where $\lambda_{\min }(\mathbf{Q})$ is the minimum eigenvalue of Q, we have

$$
\dot{V} \leq-\frac{1}{2} \lambda_{\min }(\mathbf{Q})\|\mathbf{E}\|^{2}+\left(1+g_{0} N(\varsigma)\right) \dot{\boldsymbol{\zeta}}
$$

that implies

$$
\dot{V} \leq\left(1+g_{0} N(\varsigma)\right) \dot{\zeta}
$$


Integrating Eq. (42) over $\left[t_{0}, t_{f}\right]$, one can conclude that

$$
V\left(t_{f}\right) \leq V\left(t_{0}\right)+\int_{t_{0}}^{t_{f}}() 1+g_{0} N(\varsigma) \dot{\zeta} d \tau
$$

According to Lemma 1, it is now clear that $V\left(t_{f}\right), \int_{t_{0}}^{t_{f}}\left(1+g_{0} N(\varsigma)\right) \dot{\zeta} d \tau$ and $\varsigma(t)$ are bounded in $\left[t_{0}, t_{f}\right)$. The above discussion is also true for $t_{f}=\infty$ as discussed in [7].

Integrating Eq. (41) over $\left[t_{0}, t_{f}\right]$, yields

$$
\frac{\lambda_{\min }(\mathbf{Q})}{2} \int_{t_{0}}^{t_{f}} \mathbf{E}^{T}(\tau) \mathbf{E}(\tau) d \tau \leq V\left(t_{0}\right)-V\left(t_{f}\right)+\int_{t_{0}}^{t_{f}}\left(1+g_{0} N(\varsigma)\right) \dot{\varsigma} d \tau
$$

which denotes

$$
\frac{\lambda_{\min }(\mathbf{Q})}{2} \int_{t_{0}}^{\infty} \mathbf{E}^{T}(\tau) \mathbf{E}(\tau) d \tau \leq V\left(t_{0}\right)-V(\infty)+\int_{t_{0}}^{\infty}\left(1+g_{0} N(\varsigma)\right) \dot{\zeta} d \tau
$$

Since $\int_{t_{0}}^{\infty}\left(1+g_{0} N(\varsigma)\right) \dot{\zeta} d \tau, \quad V\left(t_{0}\right)$, and $V(\infty)$ are bounded, it is concluded that $\int_{t_{0}}^{\infty} \mathbf{E}^{T}(\tau) \mathbf{E}(\tau) d \tau<\infty$. From Eqs. (32) and (41) it is derived that $\hat{\mathbf{e}}, \tilde{\mathbf{e}} \in L_{\infty}$. From the definition of $\mathbf{e}, \mathbf{x}$, and $u(t)$ it can be concluded that $\mathbf{e}, \mathbf{x}, \hat{\mathbf{x}}, u \in L_{\infty}$.

Let $\psi(t)=\mathbf{E}^{T}(t) \mathbf{E}(t)$. From Eqs. (12), and (20) since $\mathbf{E}^{T}=\left[\hat{\mathbf{e}}^{T}, \tilde{\mathbf{e}}^{T}\right]$ is bounded, it is concluded that $\dot{\mathbf{E}}(t)$ is bounded. Therefore, $\psi(t)$ is uniformly continuous and it is possible to apply the Barbalat Lemma [34] to the function $\psi(t)$ which guarantees $\lim _{t \rightarrow \infty} \psi(t)=0$, and consequently, it is deduced that $\lim _{t \rightarrow \infty} \mathbf{E}=\mathbf{0}$. Therefore, $\lim _{t \rightarrow \infty} \tilde{\mathbf{e}}=\mathbf{0}$ and $\lim _{t \rightarrow \infty} \hat{\mathbf{e}}=\mathbf{0}$. Considering that $\mathbf{e}=\hat{\mathbf{e}}+\tilde{\mathbf{e}}$ and $e_{1}=\mathbf{C}^{T} \mathbf{e}$, it can be concluded that $\lim _{t \rightarrow \infty} e_{1}=0$ that completes the proof.

Remark 5. Since the control law (29) has the sign function, chattering phenomenon, caused by the signal discontinuity, may happen when such control law is directly applied. In order to overcome this issue, a saturation function with a boundary layer $\varphi$, instead of sign function, can be considered as follows:

$$
\operatorname{sat}\left(\frac{\left(\tilde{\mathbf{e}}^{\mathbf{T}} \mathbf{P}_{2} \mathbf{B}_{c}\right)}{\varphi}\right)=\left\{\begin{array}{l}
\operatorname{sgn}\left(\frac{\left(\tilde{\mathbf{e}}^{\mathbf{T}} \mathbf{P}_{2} \mathbf{B}_{c}\right)}{\varphi}\right), \quad\left|\frac{\left(\tilde{\mathbf{e}}^{\mathbf{T}} \mathbf{P}_{2} \mathbf{B}_{c}\right)}{\varphi}\right|>1 \\
\frac{\left(\tilde{\mathbf{e}}^{\mathbf{T}} \mathbf{P}_{2} \mathbf{B}_{c}\right)}{\varphi}, \quad\left|\frac{\left(\tilde{\mathbf{e}}^{\mathbf{T}} \mathbf{P}_{2} \mathbf{B}_{c}\right)}{\varphi}\right| \leq 1
\end{array}\right.
$$

Remark 6. The presented approach is such that it can be applied to affine systems without much effort. In fact, if the system is in the affine form, the Mean Value Theorem is not required in the proof procedure. Therefore, there is no doubt that the proposed approach is also applicable to affine systems.

\section{Simulation results}

In this section, two case studies are given to demonstrate the proficiency of the proposed adaptive neural controller. The first simulation is a non-affine nonlinear system and the second one is affine. These simulations confirm that the proposed approach can deal with both non-affine and affine nonlinear uncertain systems. 
Example 1. For the first study, an academic SISO non-affine nonlinear system is considered [23]. The dynamic equations of this system are described by

$$
\begin{aligned}
& \dot{x}_{1}=x_{2} \\
& \dot{x}_{2}=-x_{1}+2 x_{2}+\frac{u}{\sqrt{|u|+0.1}}-2 x_{1}^{2} x_{2}+d(t) \\
& y=x_{1}
\end{aligned}
$$

The control goal is that the system output $x_{1}$ tracks the desired trajectory $y_{m}=0.5 \sin (t)$. The external disturbance is considered as $d_{1}(t)=0.5 \sin (2 t)$. Moreover, the design parameters are selected as $\gamma=10^{3}, k=20, \varphi=0.001$. In addition, the feedback and observer gain matrices are given by

$$
\mathbf{K}_{c}=\left[\begin{array}{ll}
144 & 24
\end{array}\right]^{T}, \quad \mathbf{K}_{o}=\left[\begin{array}{ll}
60 & 900
\end{array}\right]^{T} .
$$

The SPR filter $L_{1}^{-1}(s)$ is selected as $L_{1}^{-1}(s)=(1 / s+2)$. Besides, the positive-definite matrices $\mathbf{Q}_{1}$, and $\mathbf{Q}_{2}$ are chosen as

$$
\mathbf{Q}_{1}=\left[\begin{array}{cc}
10^{-2} & 0 \\
0 & 10^{-2}
\end{array}\right], \quad \mathbf{Q}_{2}=\left[\begin{array}{cc}
10 & 0 \\
0 & 10
\end{array}\right]
$$

In order to design the NN approximator $l=9$ nodes are taken, and the centers of basis functions are uniformly distributed in $\left[\begin{array}{ll}\hat{x}_{1} & \hat{x}_{2}\end{array}\right] \in[-2,2] \times[-2,2]$. In addition, the widths are selected as $\eta=1.8$, and the initial weights of $\mathrm{NN}$ is considered as $\boldsymbol{\Theta}_{0}=\mathbf{0}$. The simulation is carried out in MATLAB environment, and initial conditions of the system and observer are selected as

$\mathbf{x}_{0}=[-0.2,0.4]^{T}$, and $\hat{\mathbf{e}}_{0}=[0.1,0.1]^{T}$, respectively. The tracking performance of the proposed controller is illustrated in Fig. 1. Apparently, the tracking error is bounded and the proposed adaptive NN output feedback controller can excellently attain the trajectory tracking of the system output.

Fig. 2 manifests that the control signal is also bounded. The boundedness of the norm of NN weights is depicted in Fig. 3. Fig. 4 demonstrates the variation of the adaptive parameter $\varsigma$ and Nussbaum gain $N(\varsigma)$.

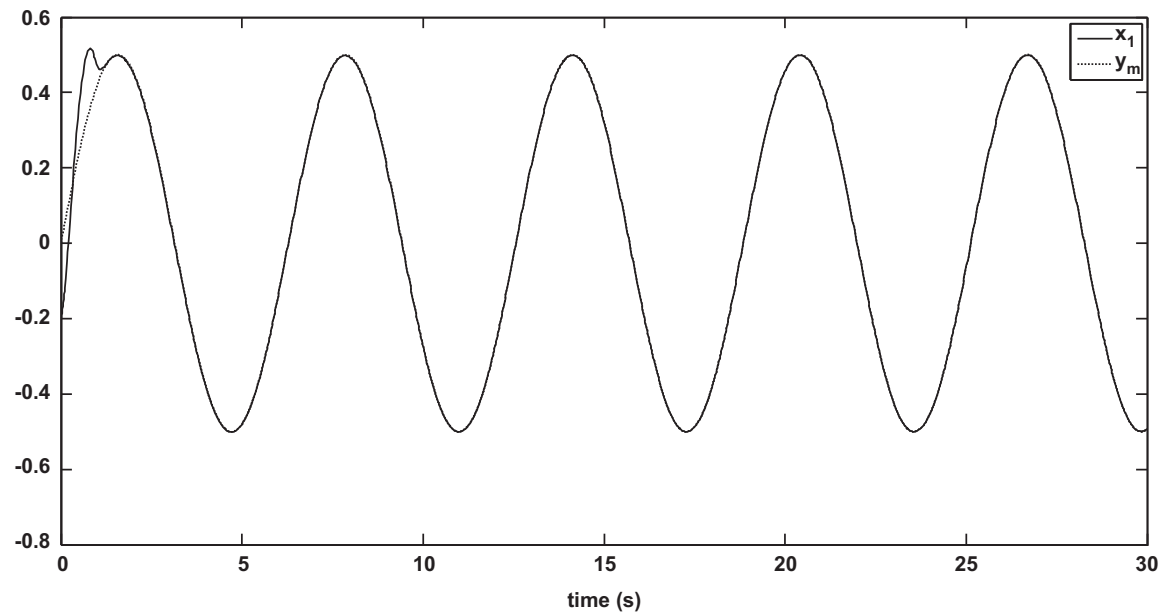

Fig. 1. Tracking performance of system output and reference signal. 


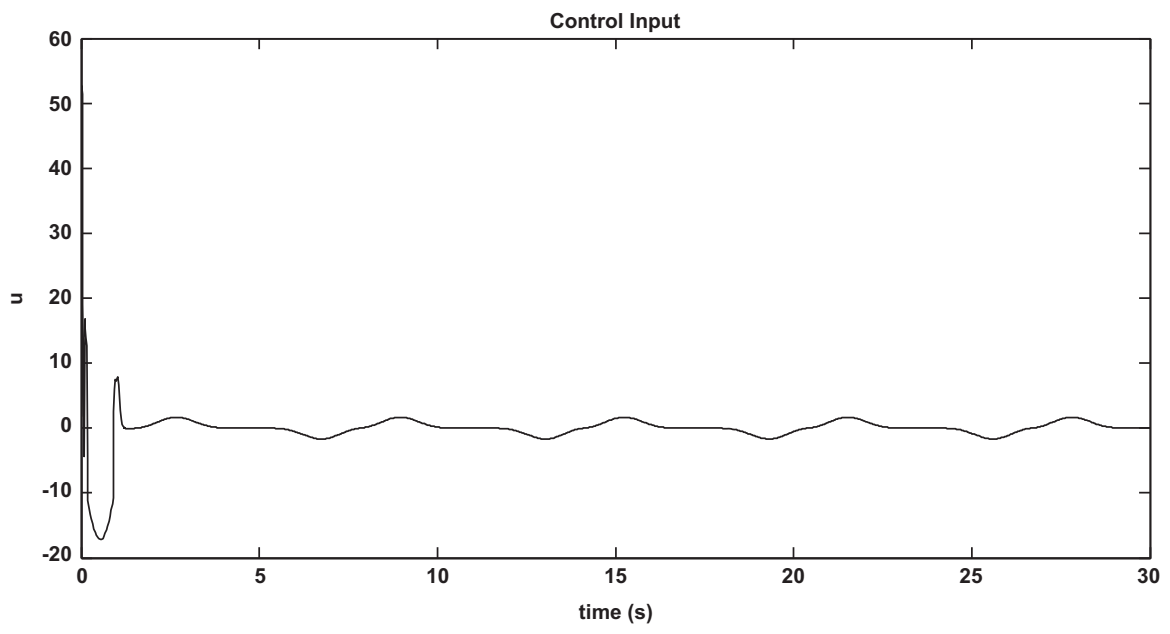

Fig. 2. Control input $u$.

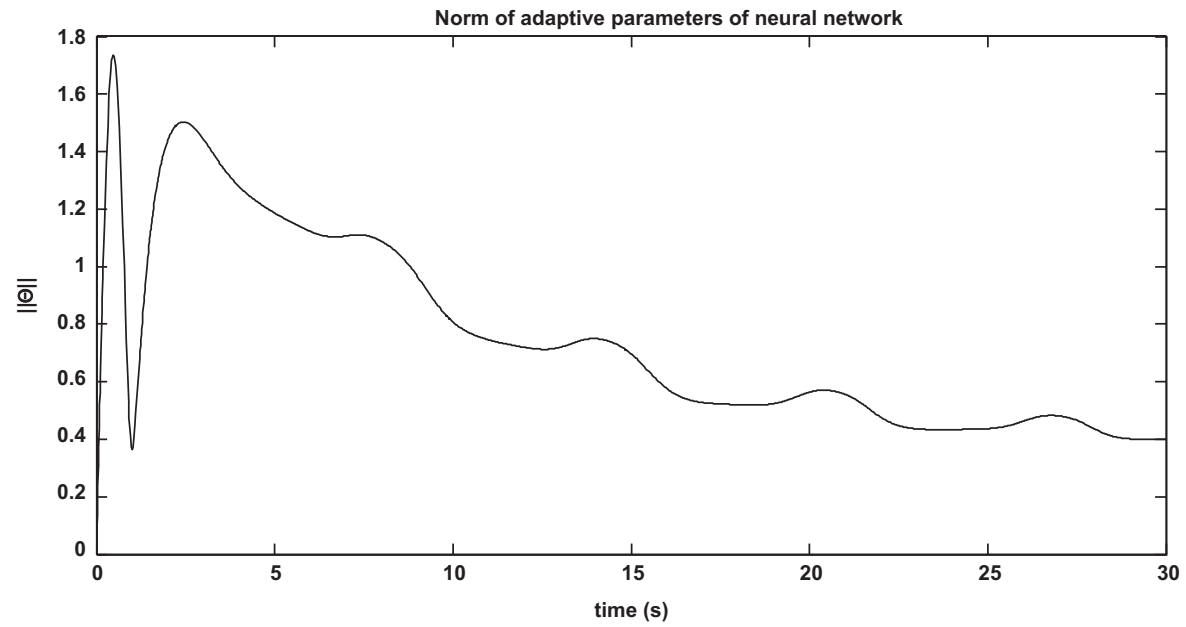

Fig. 3. The norm of adaptive weights of NN.

To show the effect of Nussbaum gain, the same simulation with the same parameters is carried out when $f(\mathbf{x}, u)$ changes to $f(\mathbf{x}, u)=-x_{1}+2 x_{2}-\frac{u}{\sqrt{|u|}+0: 1}-2 x_{1}^{2} x_{2}$. These simulations are illustrated in Fig. 5. It can be seen, the Nussbaum gain can be adapted effectively when the nonaffine function is changed while the system output tracks the desired signal successfully.

Example 2. Consider a single-link rigid manipulator. The dynamic equations of such system are given by [35]

$$
m l^{2} \ddot{q}+b \dot{q}+m \lg _{v} \cos (q)=u
$$

where $l$ and $m$ are length and mass of the link respectively, $q$ is the angular position with initial values $q(0)=0.1$ and $\dot{q}(0)=0$. The above equation can be rewritten in the form (10) with $x_{1}=$ 


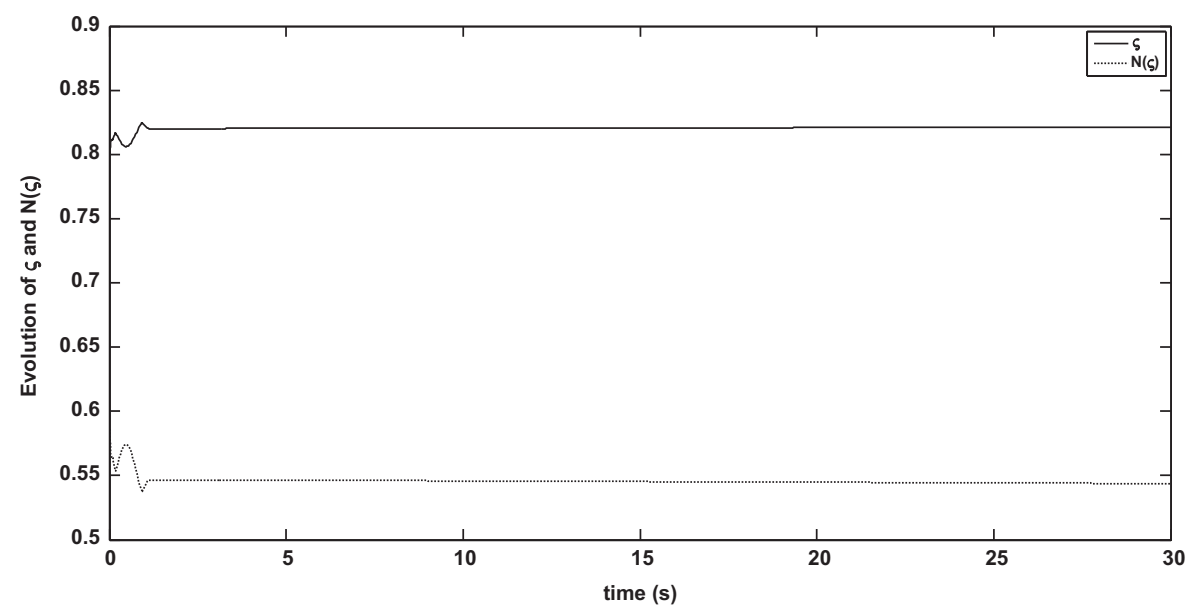

Fig. 4. The adaptive parameter $\varsigma$ (solid line) and Nussbaum gain $N(\varsigma)$ (dashed line).
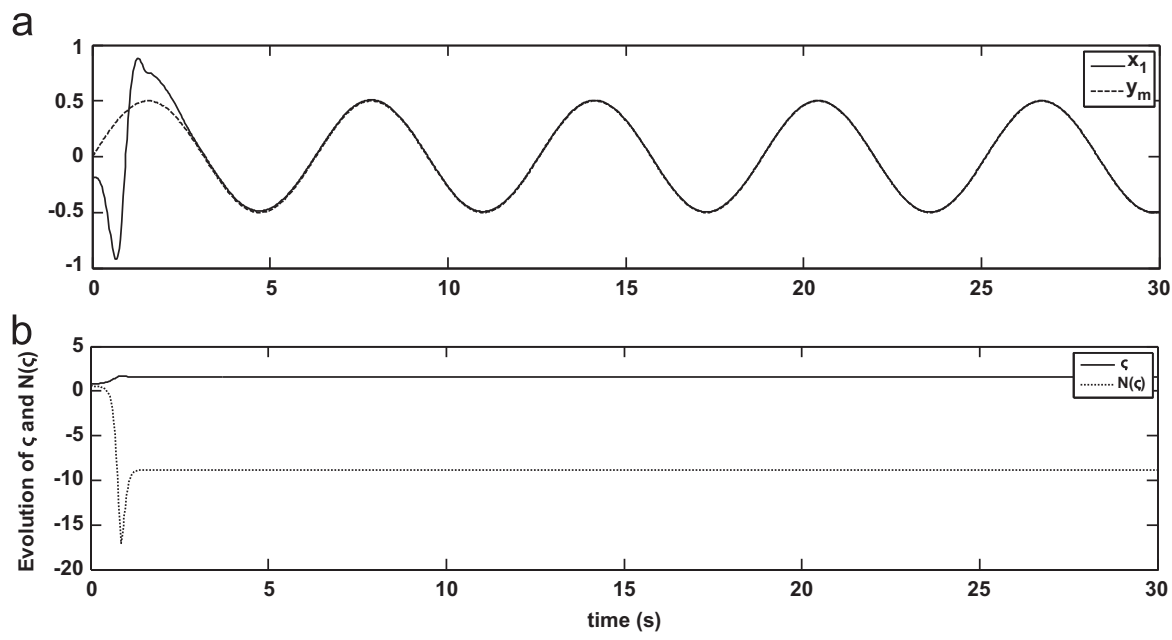

Fig. 5. (a) Tracking performance (b) $\varsigma$ (solid line) and Nussbaum gain $N(\varsigma)$ when the control gain is changed.

$x_{2}=\dot{q}, f(\mathbf{x})=\left(-\frac{b}{m l^{2}}\right) x_{2}-\left(\frac{g_{v}}{l}\right) \cos \left(x_{1}\right)$ and $g(\mathbf{x})=\left(\frac{1}{m l^{2}}\right)$. For the simulation purpose, the parameters are chosen as $m=l=b=g_{v}=1$.

The control signal is designed such that the system output tracks the desired signal $y_{m}$, which is constructed based on the following reference model:

$$
\left(\begin{array}{l}
\dot{x}_{d 1} \\
\dot{x}_{d 2}
\end{array}\right)=\left(\begin{array}{cc}
0 & 1 \\
-16 & -8
\end{array}\right)\left(\begin{array}{l}
x_{d 1} \\
x_{d 2}
\end{array}\right)+\left(\begin{array}{l}
0 \\
1
\end{array}\right) r(t)
$$

where $y_{m}=x_{d 1}$ and $r(t)$ is a pulse signal with amplitude 5 and period $10 \mathrm{~s}$. The external disturbance $d(t)=0.4 \sin (0.2 \pi t)$ is added to the system. The link mass is changed to $m=1.2$ from $t=5 \mathrm{~s}$. In this study, the controller parameters are designed as $\gamma=100, k=5, \varphi=0.001$. 


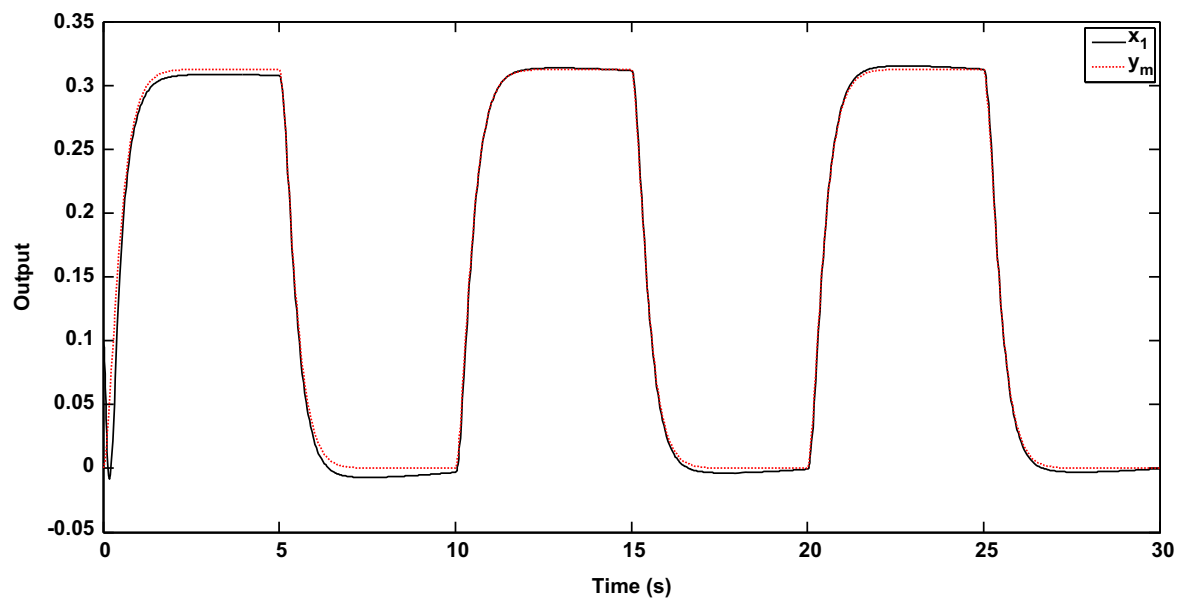

Fig. 6. Tracking performance of system output and reference signal.

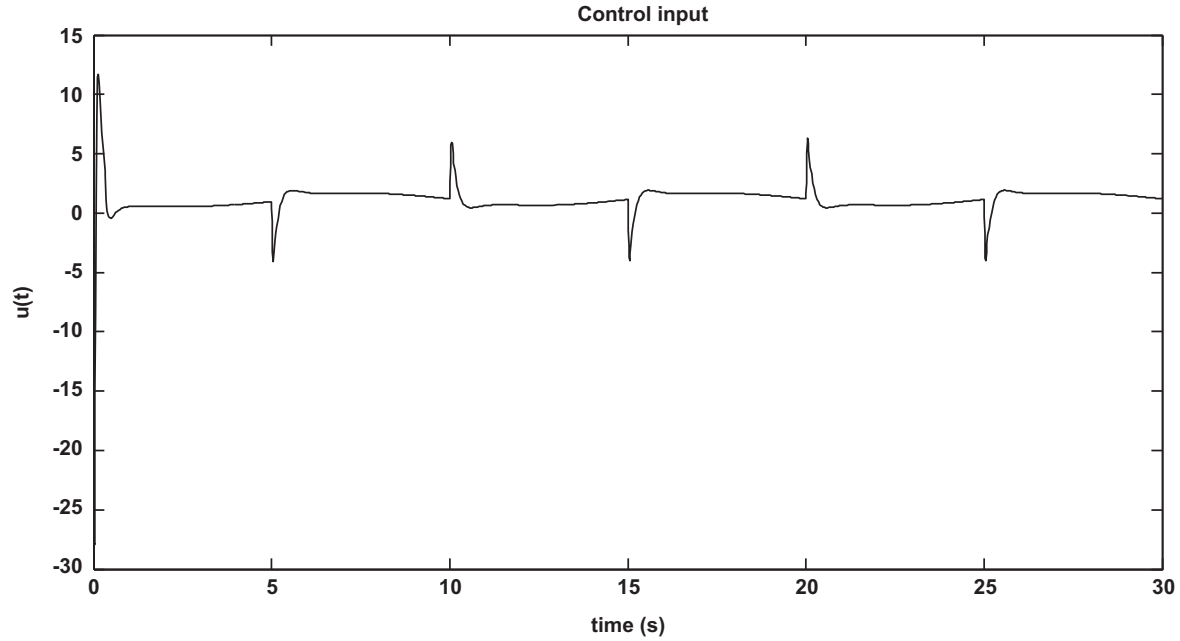

Fig. 7. Control input law $u$.

The feedback and observer gain matrices, and also matrices $\mathbf{Q}_{1}$, and $\mathbf{Q}_{2}$ are chosen as the same as the first example.

In this case study, NN parameters, except the initial elements of the weighting vector that is selected 0.1 , are constructed as similar as the previous example. $L^{-1}(s)$ are chosen as $L^{-1}=(1 / s+$ $0.5)$ to use the SPR-Lyapunov design approach. The initial condition is also considered as $\mathbf{x}_{0}=[-0.2,0.4]^{T}$, and $\hat{\mathbf{e}}_{0}=\left[-0.1,0.1^{T}\right]$. The tracking performance of the proposed controller is shown in Fig. 6. As shown in this figure, the tracking error is bounded and the desired performance is successfully achieved by the designed adaptive neural network output feedback controller.

The control signal is illustrated in Fig. 7. From these figures, it is clear that all signals, i.e. the control signal andtputs, arebounded. The variation of $\varsigma$ and the Nussbaum function $N(\varsigma)$ are shown in Fig. 8. This gureficon rms that Nussbaum gain will be automatically updated based on control direction. 


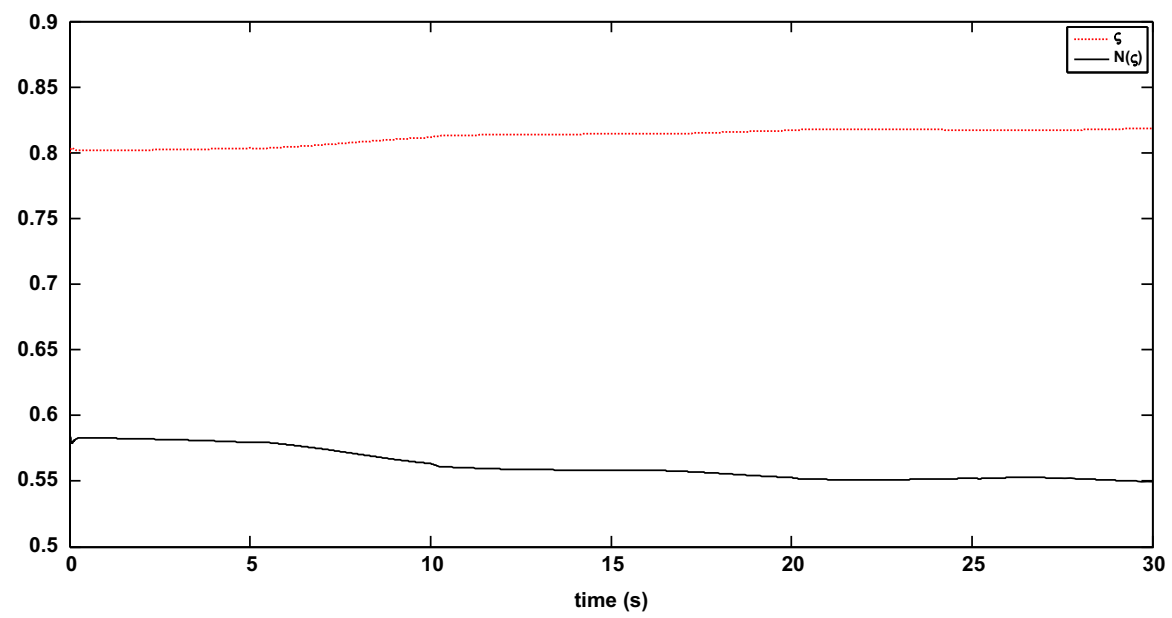

Fig. 8. The adaptive parameter $\varsigma$ (dashed line) and Nussbaum gain $N(\varsigma)$ (solid line).

\section{Conclusion}

In this paper an observer-based adaptive controller for a class of SISO non-affine nonlinear systems with unknown control gain sign has been proposed. The controller design parameters were obtained using an adaptive mechanism that exploits neural network as universal function approximator. This approach is also applicable to non-affine systems using Mean Value Theorem. In addition, Nussbaum gain technique has been utilized to deal with unknown control direction. Furthermore, in this approach, it is not necessary to assume that the system states are measurable, in other words an observer can be employed to estimate unmeasured states that are used in the controller design. Using the SPR-Lyapunov stability analysis, it has been proven that the proposed adaptive observer-based controller guarantees the asymptotic stability of the closedloop system. Finally, two simulation examples have been applied to nonlinear systems. Simulation results confirmed the effectiveness of the proposed approach when encounters both affine and non-affine nonlinear uncertain systems.

\section{References}

[1] J. Lian, S.H. Zak, Control of Uncertain Systems, Springer Handbook of Automation, 2009, pp. $199-219$.

[2] H.K. Khalil, Nonlinear Systems, third ed., Printice Hall, 2002.

[3] H.R. Karimi, Robust delay-dependent $\mathrm{H} \infty$ control of uncertain time-delay systems with mixed neutral, discrete, and distributed time-delays and Markovian switching parameters, IEEE Trans. Circuits Syst. I: Regul. Pap. 58 (2011) 1910-1923.

[4] H. Du, X. Chen, NN-based output feedback adaptive variable structure control for a class of non-affine nonlinear systems: a nonseparation principle design, Neurocomputing 72 (2009) 2009-2016.

[5] Y.J. Liu, Y.Q. Zheng, Adaptive fuzzy tracking control for a class of uncertain nonlinear systems, in: Chinese Control and Decision Conference 2009, pp. 941-944.

[6] S. Frikha, M. Djemel, N. Derbel, Observer based adaptive neuro-sliding mode control for MIMO nonlinear systems, Int. J. Control Autom. Syst. 8 (2010) 257-265.

[7] A. Boulkroune, M. Tadjine, M. M'Saad, M. Farza, Fuzzy adaptive controller for MIMO nonlinear systems with known and unknown control direction, Fuzzy Sets Syst. 161 (2010) 797-820.

[8] Y.J. Liu, Y.X. Li, Adaptive fuzzy output-feedback control of uncertain SISO nonlinear systems, Nonlinear Dyn. 61 (2010) 749-761. 
[9] B. Wang, P. Shi, H.R. Karimi, Fuzzy sliding mode control design for a class of disturbed systems, J. Frankl. Inst..

[10] X. Dong, Y. Zhao, H.R. Karimi, P. Shi, Adaptive variable structure fuzzy neural identification and control for a class of MIMO nonlinear system, J. Frankl. Inst. 350 (2013) 1221-1247.

[11] Y. Li, S. Tong, Y. Li, Observer-based adaptive fuzzy backstepping control for strict-feedback stochastic nonlinear systems with time delays, Int. J. Innov. Comput. Inf. Control 8 (2012) 8103-8114.

[12] X. Dong, Y. Zhao, Y. Xu, Z. Zhang, P. Shi, Design of PSO fuzzy neural network control for ball and plate system, Int. J. Innov. Comput. Inf. Control 7 (2011) 7091-7103.

[13] S. Sefriti, J. Boumhidi, M. Benyakhlef, I. Boumhidi, Adaptive decentralized sliding mode neural network control of a class of nonlinear interconnected systems, Int. J. Innov. Comput. Inf. Control 9 (2013) 2941-2947.

[14] Y.J. Liu, S.C. Tong, W. Wang, Y.M. Li, Observer-based direct adaptive fuzzy control of uncertain nonlinear systems and its applications, Int. J. Control Autom. Syst. 7 (2009) 681-690.

[15] H.X. Li, S. Tong, A hybrid adaptive fuzzy control for a class of nonlinear MIMO systems, IEEE Trans. Fuzzy Syst. 11 (2003) 24-34.

[16] S. Labiod, T.M. Guerra, Direct adaptive fuzzy control for a class of MIMO nonlinear systems, Int. J. Syst. Sci. 38 (2007) 665-675.

[17] R. Ordónez, K.M. Passino, Stable multi-input multi-output adaptive fuzzy/neural control, IEEE Trans. Fuzzy Syst. 7 (1999) 345-353.

[18] T. Shaocheng, C. Bin, W. Yongfu, Fuzzy adaptive output feedback control for MIMO nonlinear systems, Fuzzy Sets Syst. 156 (2005) 285-299.

[19] M.M. Arefi, M.R. Jahed-Motlagh, Observer-based adaptive neural control of uncertain MIMO nonlinear systems with unknown control direction, Int. J. Adapt. Control Signal Process. 27 (2013) 741-754.

[20] Y.G. Leu, T.T. Lee, W.Y. Wang, Observer-based adaptive fuzzy-neural control for unknown nonlinear dynamical systems, IEEE Trans. Syst. Man Cybern. B Cybern. 29 (1999) 583-591.

[21] Y.C. Chang, Robust tracking control for nonlinear MIMO systems via fuzzy approaches, Automatica 36 (2000) 1535-1545.

[22] R.D. Nussbaum, Some remarks on a conjecture in parameter adaptive control, Syst. Control Lett. 3 (1983) $243-246$.

[23] M.M. Arefi, M.R. Jahed-Motlagh, Observer-based adaptive neural control for a class of nonlinear non-affine systems with unknown gain sign, in: 18th IFAC World Congress, 2011, pp. 2644-2649.

[24] Y.J. Liu, Z.F. Wang, Adaptive fuzzy controller design of nonlinear systems with unknown gain sign, Nonlinear Dyn. 58 (2009) 687-695.

[25] J. Rubió-Massegú, J.M. Rossell, H. Karimi, F. Palacios-Quiñonero, Static output-feedback control under information structure constraints, Automatica 49 (2012) 313-316.

[26] Y. Yin, P. Shi, F. Liu, H.R. Karimi, Observer-based $H_{\infty}$ control on stochastic nonlinear systems with time-delay and actuator nonlinearity, J. Frankl. Inst. 350 (2013) 1388-1405.

[27] H.R. Karimi, Passivity-based output feedback control of Markovian jump systems with discrete and distributed time-varying delays, Int. J. Syst. Sci. 44 (2013) 1290-1300.

[28] H.X. Li, S. Tong, A hybrid adaptive fuzzy control for a class of nonlinear MIMO systems, IEEE Trans. Fuzzy Syst. 11 (2003) 24-34.

[29] Q. Zhou, P. Shi, S. Xu, H. Li, Observer-based adaptive neural network control for nonlinear stochastic systems with time delay, IEEE Trans. Neural Networks Learn. Syst. 24 (2013) 71-80.

[30] T. Shaocheng, L. Yongming, S. Peng, Observer-based adaptive fuzzy backstepping output feedback control of uncertain MIMO pure-feedback nonlinear systems, IEEE Trans. Fuzzy Syst. 20 (2012) 771-785.

[31] S. Labiod, T.M. Guerra, Adaptive fuzzy control of a class of SISO nonaffine nonlinear systems, Fuzzy Sets Syst. 158 (2007) 1126-1137.

[32] J.H. Park, S.H. Huh, S.H. Kim, S.J. Seo, G.T. Park, Direct adaptive controller for nonaffine nonlinear systems using self-structuring neural networks, IEEE Trans. Neural Netw. 16 (2005) 414-422.

[33] Y. Xudong, J. Jingping, Adaptive nonlinear design without a priori knowledge of control directions, IEEE Trans. Autom. Control 43 (1998) 1617-1621.

[34] R. Marino, P. Tomei, Nonlinear Control Design: Geometric, Adaptive and Robust, Prentice Hall, London, 1995.

[35] M. Chemachema, Output feedback direct adaptive neural network control for uncertain SISO nonlinear systems using a fuzzy estimator of the control error, Neural Netw. 36 (2012) 25-34. 